\title{
Outskirts of Local Group Dwarf Galaxies Revealed by Subaru Hyper Suprime-Cam
}

\section{Yutaka Komiyama}

National Astronomical Observatory of Japan, 2-21-1, Osawa, Mitaka, Tokyo 181-8588, Japan email: komiyama@subaru.naoj.org

\begin{abstract}
Local Group galaxies are important targets since their stellar populations can be resolved, and their properties can be investigated in detail with the help of stellar evolutionary models. The newly-built instrument for the $8.2 \mathrm{~m}$ Subaru Telescope, Hyper Suprime-Cam (HSC), which has a 1 Giga pixel CCD camera with 1.5 degrees field of view, is the best instrument for observing Local Group galaxies. We have carried out a survey for Local Group dwarf galaxies using HSC aiming to shed light on the outskirts of these galaxies. The survey covers target galaxies out beyond the tidal radii down to a depth unexplored by previous surveys. Thanks to the high spatial resolution and high sensitivity provided by the Subaru Telescope, we are able to investigate properties such as spatial distribution and stellar population from the very center of galaxies to the outskirts. In this article, I will show results for the dwarf irregular galaxy NGC 6822 and the dwarf spheroidal galaxy Ursa Minor.
\end{abstract}

Keywords. Local Group, dwarf galaxy, star formation, structure

\section{The Dwarf Irregular Galaxy NGC 6822}

NGC 6822 is an active star-forming dwarf irregular galaxy located at 500kpc from the Milky Way. The structure of NGC 6822 is known to be intriguing: an old $(>>1$ Gyr) stellar population shows a round and smooth distribution (e.g., Battinelli et al. $2006)$, while, in contrast, the young $(<500 \mathrm{Myr})$ stellar population shows an elongated distribution, which traces the distribution of neutral hydrogen very well (e.g., Komiyama et al. 2003). The elongated distribution of the young stellar population is suggested to be produced owing to the interaction between the main body of NGC 6822 and HI cloud to the north-west over the last several $100 \mathrm{Myr}$ (de Blok \& Walter 2000).

We carried out a deep survey of NGC 6822. A single pointing of HSC covers the tidal radius of NGC 6822 as well as the entire HI structure and our deep broad-band data clearly show the previously characterized stellar distribution. We also observed this galaxy with NB0656 narrow-band filter which samples $\mathrm{H} \alpha$ emission, aiming to shed light on ongoing star-forming activity characterized by $\mathrm{H} \alpha$ emission. The total integration time of NB0656 data is 1.25 hours and the data reach to $\sim 26 \mathrm{mag}(\mathrm{AB})$. As clearly shown in Fig. 1, our high resolution continuum subtracted $\mathrm{H} \alpha$ image elucidates the detailed structures of $\mathrm{H} \alpha$ emission regions at the center of NGC 6822. In addition, many faint but significant $\mathrm{H} \alpha$ emission regions are found in the outskirts of this galaxy, extending the pioneering work by de Blok \& Walter (2006).

We compared the $\mathrm{H} \alpha$ image with an FUV image obtained by GALEX. Since FUV is a direct measure of OB stars embedded in HII regions, a good correlation between $\mathrm{H} \alpha$ emission regions and FUV sources is expected. The comparison shows that the majority of $\mathrm{H} \alpha$ emission regions are coincident with FUV sources. It is also found that the sizes of some exceptions (i.e., $\mathrm{H} \alpha$ emission without FUV sources) are likely to be smaller. 


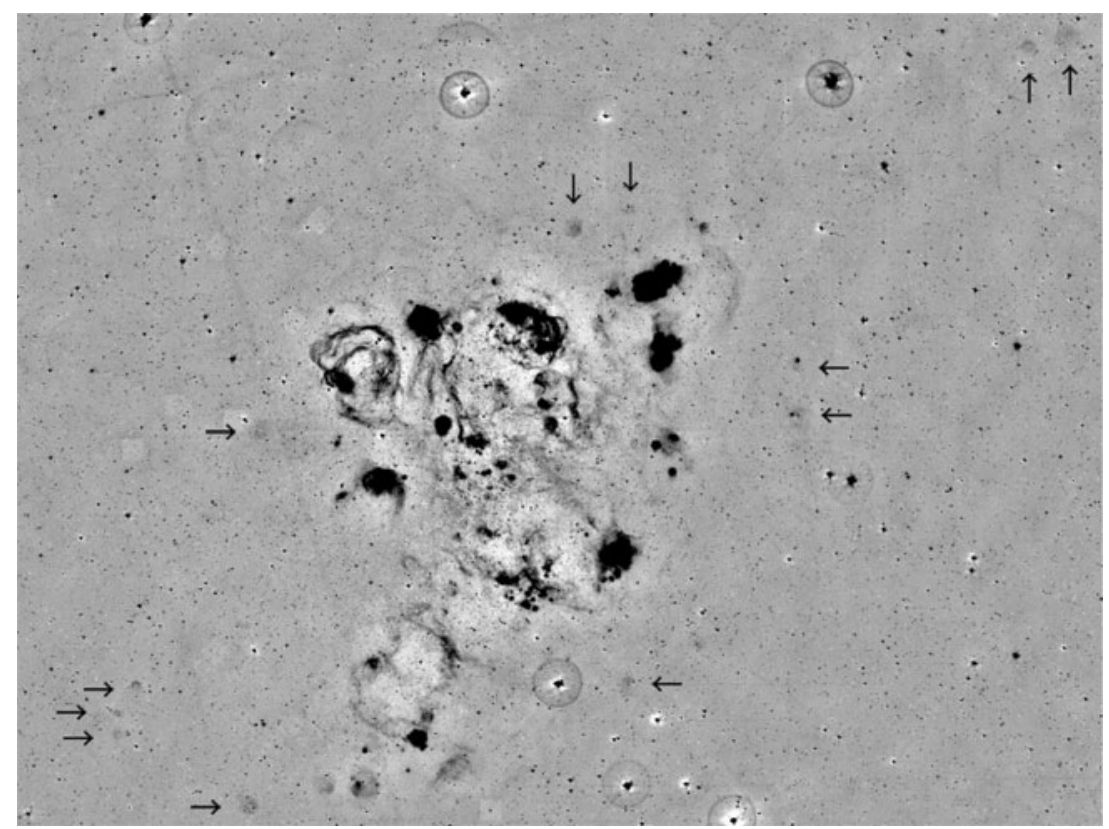

Figure 1. Continuum subtracted $\mathrm{H} \alpha$ image of NGC 6822. The color scheme of the image is inverted for clarity. Faint $\mathrm{H} \alpha$ emission regions are indicated by arrows.

We also compared our $\mathrm{H} \alpha$ image with high-resolution map of neutral hydrogen published by de Blok \& Walter (2000). The distribution of neutral hydrogen around NGC 6822 shows complicated morphology (see Fig. 1 of de Blok \& Walter 2000); a north-west cloud, giant hole at the east of main body, and arm to the south-east. It is found that 1) $\mathrm{H} \alpha$ emission regions are always found where neutral hydrogen is abundant, but 2) they are not always coincident with the peak position of neutral hydrogen, 3) the giant hole is surrounded by a number of $\mathrm{H} \alpha$ emission regions, and 4) no significant $\mathrm{H} \alpha$ emission region is detected for the south-east HI arm.

\section{The Dwarf Spheroidal Galaxy Ursa Minor (UMi)}

Since its discovery in mid-20th century, the Ursa Minor dwarf spheroidal galaxy has been observed extensively and suggested to be a dark matter dominated galaxy (M/L within $r_{1 / 2}=290 \mathrm{M}_{\odot} / \mathrm{L}_{\odot}$; Pace et al. 2014). As the observational clues have accumulated, it began to appear that UMi is not a simple system: UMi is likely to host hidden substructures in the main body (from radial velocity analysis; e.g., Pace et al. 2014) and extra-tidal structures (from radial profile analysis; Palma et al. 2003), indicating that even a small system like UMi may have had a complicated evolutionary history. Therefore, UMi is an important target to better understand how dwarf glaxies have evolved.

We obtained 4 pointings around UMi to explore the center of UMi to far beyond the tidal radius. Short exposure frames (90 seconds in both $g$ and $i$ bands) provide accurate photometry of stars over a wide range of brightnesses, from the tip of red giant branch (RGB) at $i \simeq 16$ mag to the main sequence (MS) turn off point at $i \simeq 23$ mag. The color magnitude diagrams (CMD) shows a narrowly defined stellar sequence from tip of RGB to MS, suggesting a homogeneous population in UMi. We investigated the CMD in a series of elliptical annuli and find that the narrow stellar sequence persists beyond the tidal radius, up to $\sim 2$ tidal radii. 

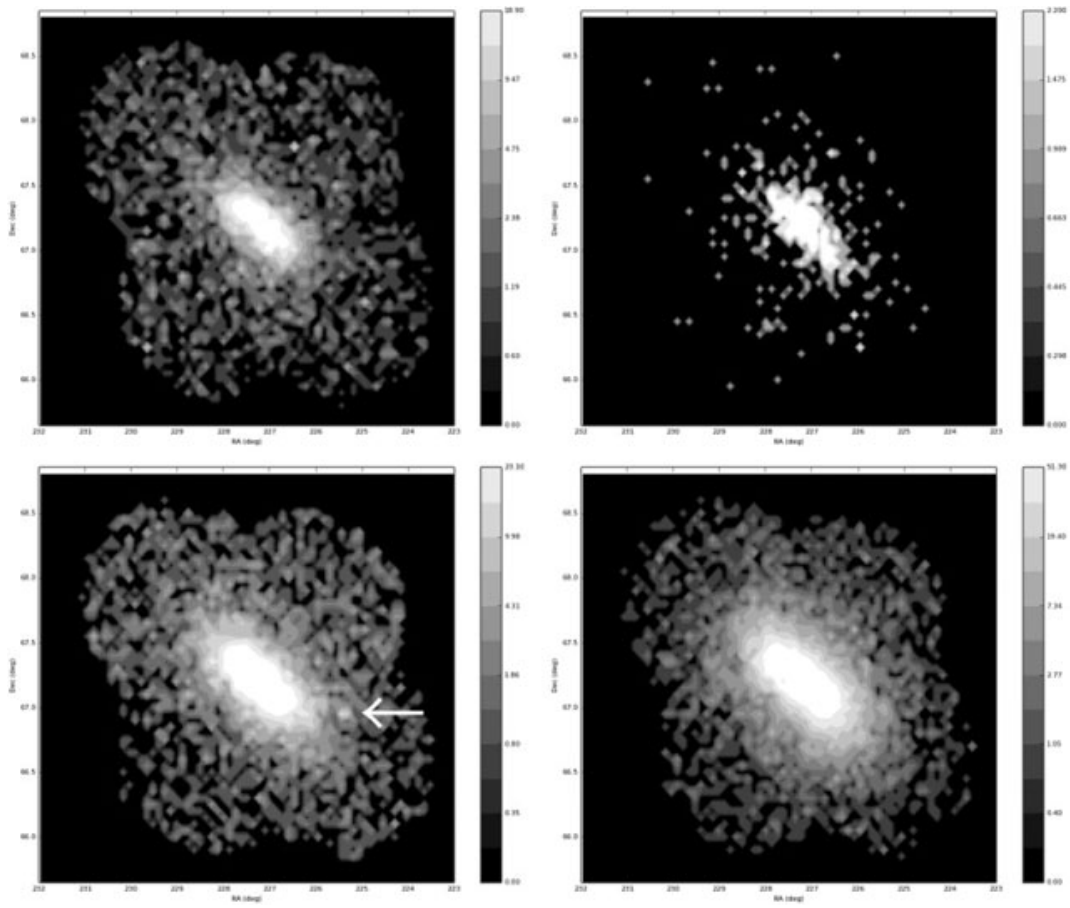

Figure 2. Density maps of RGB (top-left), BHB (top-right), SGB (bottom-left) and MS (bottom-right) stars in the Ursa Minor field. Muñoz et al. star cluster is marked with an arrow.

Based on the position in the CMD, RGB, blue horizontal branch (BHB), sub-giant branch (SGB; $22<i<23$ ) and MS stars $(23<i<24.5)$ are selected and spatial distributions of each component are investigated. From the density maps plotted in Fig. 2, we conclude that no significant substructure is found for the outskirts of UMi except for the west side of UMi; a significant peak is found in the figures for SGB and MS. However, this structure is identified to be a known star cluster which is located between Milky Way and UMi (Muñoz et al. 2012).

In addition to the broad-band data, we have narrow-band (NB) data observed with NB0515 filter which is equivalent to the DDO51 filter used by Palma et al. (2003) and samples the Mgb absorption line at 515nm. Using NB0515 data, we can discriminate RGB stars belonging to UMi from Milky Way M dwarfs with much higher reliability. We investigated the spatial distribution of more probable UMi RGB stars and found that the NB-selected RGB stars seem to be extended towards north-east direction. Similar distribution is also reported by Palma et al. (2003), suggesting that some diffuse substructure may exist in north-east direction of UMi.

\section{References}

Battinelli, P., Demers, S., \& Kunkel, W. E. 2006, A\&A, 451, 99

de Blok, W. J. G. \& Walter, F. 2000, ApJL, 537, L95

de Blok, W. J. G. \& Walter, F. 2006, AJ, 131, 343

Muñoz, R. R., Geha, M., Côte, P., et al. 2012, ApJL, 753, L15

Komiyama, Y., Okamura, S., Yagi, M., et al. 2003, ApJL, 590, L17

Pace, A. B., Martinez, G. D., Kaplinghat, M., \& Muñoz, R. R. 2014, MNRAS, 442, 1718

Palma, C., Majewski, S. R., Siegel, M. H., et al. 2003, AJ, 125, 1352 